\title{
Integrating Gene Mutation Mutual Exclusion Information Into Radiomics Algorithms to Improve Gene Mutation Prediction in Non-small Cell Lung Cancer
}

\author{
Jingyi Wang \\ Xijing Hospital \\ Xing Lv \\ Xijing Hospital \\ Xin Cao \\ Northwest University \\ Weicheng Huang \\ Northwest University

\section{Zhiyong Quan} \\ Xijing Hospital
}

Guiyu Li

Xijing Hospital

Xiaohu Zhao

Xijing Hospital

Yirong Wang

Xijing Hospital

Zhaojuan Xie

Xijing Hospital

Yuhao Yan

Xijing Hospital

Yuhua Gao

Xijing Hospital

Xiang Li

Xijing Hospital

Wenhui Ma

Xijing Hospital

\section{Weidong Yang}

Xijing Hospital

Fei Kang ( $\nabla$ fmmukf@qq.com )

Fourth Military Medical University https://orcid.org/0000-0003-0267-7819 
Jing Wang

Xijing Hospital

\section{Research Article}

Keywords: PET/CT, mutation mutual exclusion information, radiomics, EGFR, KRAS

Posted Date: October 6th, 2021

DOl: https://doi.org/10.21203/rs.3.rs-942839/v1

License: (c) (1) This work is licensed under a Creative Commons Attribution 4.0 International License. Read Full License 


\section{Abstract}

Purpose Gene mutations are mutually exclusive in non-small cell lung cancer (NSCLC). Using EGFR and KRAS as examples, this study aims to assess the significance of mutation mutual exclusion information in the optimization of radiomics algorithms for predicting gene mutation.

Methods We retrospectively analyzed 161 NSCLC patients with ${ }^{18}$ F-FDG PET/CT scans and EGFR and KRAS gene mutation data. Patients were randomly divided into training and testing cohorts. The Pyradiomics toolkit was used for radiomics feature extraction. Based on these features, radiomics score (RS) models were developed for predicting KRAS mutations using the gradient boosting decision tree (GBDT) algorithm. Furthermore, to investigate the value of adding mutation mutual exclusion information, a composite model combining PET/CT RS and EGFR mutation status was developed using logistic regression. The area under the curve (AUC), specificity, sensitivity, and accuracy were calculated for model performance evaluation in the training and test cohorts. To test the generalizability of this optimization method, models for predicting EGFR mutation were established in parallel, with or without adding KRAS gene mutation information.

Results Compared with CT, the PET/CT based RS model exhibited higher AUC (KRAS: 0.792 vs 0.426; EGFR: 0.786 vs 0.644 ). By integrating EGFR mutation information into the PET/CT RS model, the AUC, accuracy, and specificity for predicting KRAS mutations were all elevated in the test cohort $(0.928,0.857$, 0.897 vs $0.792,0.755,0.769)$. Conversely, the composite model for predicting EGFR mutations could also be optimized by adding KRAS gene mutation information (AUC, accuracy, and specificity: $0.877,0.776$, 0.700 vs $0.786,0.694,0.567)$. By adding EGFR and KRAS exclusive mutation information, respectively, the composite model corrected $55.4 \%$ and $30.7 \%$ false positive cases produced by the PET/CT RS model in the test cohort, without sacrificing sensitivity.

Conclusion Integrating the mutation status of a known gene is a potential method to optimize radiomics models for predicting another gene mutation. This method may help predict unconventional gene mutations when the second biopsy is clinically difficult to carry out.

\section{Introduction}

As of 2020, lung cancer is the leading cause of cancer deaths in the world [1-3]. Non-small cell lung cancer (NSCLC) is the main type of lung cancer, accounting for about $85 \%$ of the total lung cancer cases $[4,5]$. Anti-cancer therapies targeting the Epidermal Growth Factor Receptor-Tyrosine Kinase Inhibitor (EGFR-TKI) pathway are widely used in the clinic, effectively prolonging survival of NSCLC patients [6-8]. Therefore, determining the mutation status of molecular targets like EGFR-TKI is a prerequisite to identify potentially effective targeted therapies and to avoid treatments that are unlikely to provide clinical benefit [9].

However, several international guidelines only agree on the need for testing conventional mutations (EGFR, BRAF, ALK, and ROS1) in advanced NSCLC [10]. In instances where these conventional mutations 
were absent, knowing the mutation status of an unconventional gene (e.g. KRAS, RET, and NTRK) is particularly in demand for choosing newly approved targeted drugs [11-15]. Risking a second biopsy or conducting expensive next-generation sequencing (NGS) increases the difficulty of clinical implementation $[10,16-18]$. Therefore, there is an urgent need to develop a non-invasive method to determine gene mutation status.

With recent advances in artificial intelligence, radiomics has been widely used in the detection of gene mutations [19-21], and the performance of these radiomics models has been subject to continuous optimization [22]. To improve the accuracy in predicting gene mutations, previous studies integrated various clinical information, including smoking history, radiographic features, and serum tumor markers into radiomics models [21, 23-25]. However, because of the weak theoretical connection between the above clinical information and genetic mutations, a better optimization method may be potentially achieved by integrating more directly relevant genetic information.

Multiple studies have shown that both conventional and unconventional gene mutations, such as EGFR, KRAS, ROS1, BRAF, METex14 skipping mutations, RET rearrangements, and ALK are mutually exclusive in lung cancer $[26,27]$. Therefore, the known genetic mutation status of one gene is theoretically directly relevant to another. This mutation mutual exclusion information may be useful in optimizing the accuracy of radiomics models.

Using EGFR and KRAS as an example, the current study aims to verify whether knowledge of the conventional EGFR gene mutation can improve the accuracy of the radiomics model for predicting an unconventional KRAS gene mutation, based on ${ }^{18} \mathrm{~F}$-FDG PET/CT multimodality imaging data. Furthermore, to test the generalizability of this optimization method, models for predicting EGFR mutations were also established in parallel, with or without adding the mutation information of KRAS.

\section{Materials And Methods}

\section{Patients}

We retrospectively analyzed the PET/CT images of NSCLC patients diagnosed pathologically in Xijing Hospital from 2016 to 2020. A total of 161 NSCLC patients were screened, all of which received EGFR and KRAS genetic testing at the primary site of lung cancer. It should be noted that since KRAS is not a conventional recommended target for testing in ESMO, CAP/IASLC/AMP, and Pan-Asian guidelines, patients undergoing additional sequencing are rare in current clinical practice [10]. Inclusion criteria were: (1) confirmation of NSCLC by pathology or cytology; (2) having undergone ${ }^{18} \mathrm{~F}-\mathrm{FDG}$ PET/CT imaging; (3) primary lesion diameter $>1 \mathrm{~cm}$; (4) no history of other malignant tumors. Exclusion criteria were: (1) having received radiotherapy or chemotherapy within 2 weeks before PET/CT examination; (2) poor $\mathrm{PET} / \mathrm{CT}$ image quality. This retrospective study was approved by the Medical Ethics Committee of Xijing Hospital (Approval No. KY20173008-1). 
All cases were randomly assigned in a 7:3 ratio to the training cohort $(n=112)$ or testing cohort $(n=49)$. All cases in the training cohort were used to train the predictive model, while cases in the testing cohort were used to independently evaluate the model's performance.

\section{${ }^{18}$ F-FDG PET/CT Imaging}

All patients received ${ }^{18} \mathrm{~F}$-FDG PET/CT scans on the same equipment (Biograph 40, Siemens), following a standard clinical protocol [28]. Briefly, patients were required to fast for $>6$ hours before the scan and exhibit blood glucose control within $7.8 \mathrm{mmol} / \mathrm{L}$. CT parameters were $100 \mathrm{kV}, 110 \mathrm{mAs}, 0.5 \mathrm{~s}$ rotation time, $5.0 \mathrm{~mm}(24 \times 1.2 \mathrm{~mm})$ slice thickness, $700 \mathrm{~mm}$ field of view, and $512 \times 512$ matrix. For PET scanning, $4.44 \sim 5.55 \mathrm{MBq} / \mathrm{kg}$ of ${ }^{18} \mathrm{~F}-\mathrm{FDG}$ was injected. Scanning was initiated $60 \mathrm{~min}$ after tracer administration, with 3 min scans per bed position. PET and CT images were reconstructed using an ordered-subsets expectation-maximization algorithm with four iterations and eight subsets [29-31].

\section{Tumor segmentation, feature extraction, and selection}

Following previously published methods [19, 22, 25, 32-34], all regions of interest (ROI) were defined for PET and CT images by two experienced nuclear medicine physicians using MITK (Medical Imaging interaction Tool Kit v2018.04.2) software. CT images were delineated manually on the lung window $(\mathrm{WL}=-500 \mathrm{HU}, \mathrm{WW}=1500 \mathrm{HU}), \mathrm{PET}$ images were delineated by segmentation threshold and region-growing 3D segmentation and then manually adjusted.

Following published methods [33, 35-37], the Pyradiomics software package was used to extract radiomics features, with a total of 4306 features extracted. Missing values were replaced with the median. All data were subjected to standardized data preprocessing. Feature selection was performed using univariate and multivariate analyses with a stepwise selection method. First, Mann-Whitney U tests were performed to identify features with a statistical threshold of $p<0.05$. Pearson and Spearman correlation analysis was used to analyze the correlation between various features. For pairs of features with an $r$ value $>0.8$, only one was kept. Recent studies showed that the gradient boosting decision tree (GBDT) algorithm is better than the commonly used least absolute shrinkage and selection operator (LASSO) algorithm, so we employed the GBDT algorithm to select optimal features in the current study [37].

\section{Model establishment, comparison, and evaluation}

CT radiomics score (RS) and PET/CT RS logistic models were built from the established optimal feature subsets of the training cohort. A better performing RS was then selected from a multimodal RS model combining EGFR or KRAS mutation information to develop the composite model. Calibration curves were plotted to evaluate the goodness of fit of the prediction model. Performances of the composite model and RS were subsequently compared in the training and test cohorts. Performance parameters included the area under the curve (AUC), accuracy, sensitivity, and specificity. AUCs of the three models were 
compared using the DeLong test. Decision curve analysis was performed to further compare the clinical benefit of the RS and composite model.

\section{Statistical analysis}

All statistical analyses were performed using R software version 3.5.1 and Python software version 3.5.6. Mann-Whitney U tests were used to compare continuous variables, while Chi-squared tests were used to compare differences in categorical variables. A p-value $<0.05$ indicated statistical significance.

\section{Results}

\section{Patient Characteristics}

The study objective was to verify whether limited mutation information and mutation mutual exclusivity logic can be used to make more efficient radiomics predictions for other gene mutations. This study is divided into two parts (Part I and Part II): Part I establishes three models (CT RS, PET/CT RS, and superior RS combined with EGFR) to verify whether adding EGFR mutation information can improve RS model accuracy for predicting KRAS mutations; Part II establishes three models (CT RS, PET/CT RS, and superior RS combined with KRAS) to verify whether adding KRAS mutation information can improve RS model accuracy for predicting EGFR mutations.

Clinical characteristics of the 161 patients in Part I and Part II are shown in Table 1 and Table 2, respectively. There were no significant differences in subjective characteristics between the training and test cohorts in Part I and Part II, including for age $(p=0.942 / 0.456)$, gender $(p=0.124 / 0.323)$, smoking $(p=0.087 / 0.467), \operatorname{CEA}(p=0.177 / 0.500), \operatorname{EGFR}(p=0.691$, Part I), and KRAS $(p=0.911$, Part II). The lack of differences indicates that the random grouping in this study was reasonable. Chi-square testing revealed that EGFR mutation was a significant predictor of KRAS mutation ( $p<0.001$ in training cohort, $p=0.01$ in test cohort), and KRAS mutation was a significant predictor of EGFR mutation $(p<0.001$ in training cohort, $p=0.014$ in test cohort).

\section{Feature selection and RS establishment}

In the study predicting KRAS mutations, 8 features were included in the CT RS model and 9 features were included in the PET/CT RS model (6 CT and 3 PET features). In the study predicting EGFR mutations, 10 features were included in the CT RS model and 14 features were included in the PET/CT RS model (5 CT and 9 PET features). The PET/CT RS was significantly different between patients with KRAS mutations and those with wild type KRAS ( $p<0.001$ in training cohort, $p=0.005$ in test cohort). See Supplementary Tables S1 for detailed information. The PET/CT RS was significantly different between patients with EGFR mutations and those with wild type EGFR ( $p<0.001$ in training cohort, $p=0.001$ in test cohort). See Supplementary Tables S2 for detailed information. PET/CT RS for each patient in the two cohorts is displayed in Fig. 1. RS formulas are displayed in the supplementary materials. 
In the test cohort, the AUC, sensitivity, and accuracy of the PET/CT RS model were superior to the CT RS model (KRAS - AUC: 0.792 vs 0.426, sensitivity: 0.700 vs 0.300, accuracy: 0.755 vs 0.673; EGFR - AUC: 0.786 vs 0.644 , sensitivity: 0.895 vs 0.737 , accuracy: 0.694 vs $0.592 ;$ Fig. 2$)$. Therefore, we established composite models combining PET/CT RS and EGFR or KRAS mutation information. The composite model performed better than the PET/CT RS model in both parts of the study, with an AUC of 0.961 (95\% $\mathrm{Cl}[0.928,0.989])$ in the training cohort and $0.928(95 \% \mathrm{Cl}[0.846,0.991])$ in the test cohort of Part I, and an AUC of $0.926(95 \% \mathrm{Cl}[0.885,0.962])$ in the training cohort and $0.877(95 \% \mathrm{Cl}[0.794,0.951])$ in the test cohort of Part II. The Delong test p-value map is shown in Fig. 3. Specific comparison data of the three models, including AUC, sensitivity, specificity, and accuracy, are shown in Table 3 and Table 4.

The false positive rate (FPR) of the composite model (10.3\%) was significantly decreased compared to the PET/CT RS model (23.1\%) in the Part I test cohort, and was decreased from $43.3 \%$ to $30 \%$ in the Part II test cohort, and neither generated additional false negative error. In the test cohort, the composite model corrected $55.4 \%$ and $30.7 \%$ false positive errors generated by the PET/CT RS model in the Part I and Part Il studies, respectively. Two nomograms were created for further clinical use in predicting KRAS and EGFR mutation status (Fig. 4a and Fig. 5a). The nomogram calibration curve is presented in Fig. 4b and c, 5b and $\mathrm{c}$, which indicated good consistency between the predicted and actual values.

\section{Decision curve analysis}

To evaluate the clinical usefulness of the established radiomics models, decision curves were drawn (Fig. $6 \mathrm{a}$ and $\mathrm{b}$ ). The composite model had higher net benefit than CT and PET/CT RS models, and the threshold probability reached $<60 \%$ in both Part I and II.

\section{Discussion}

In this study, we observed two main findings. First, we found that adding information of a known gene mutation is a new method to predict mutation status for an additional gene, which was mutually validated for predicting both KRAS and EGFR mutations in lung cancer. Second, compared with the PET/CT RS model, the combination of PET/CT RS and mutation mutual exclusion information improved the accuracy and AUC for predicting mutations without significantly affecting sensitivity. These findings can improve the ability of radiomics to predict gene mutations, and may avoid second biopsies for determining unconventional gene mutation status in patients at risk.

Since the introduction of radiomics in 2012, it has been widely used to predict genetic mutations. Much effort has been made by researchers to optimize the ability of radiomics to predict gene mutations. For example, in a study by Zhang et al. on the prediction of EGFR mutations in lung cancer using radiomics, a complex model combining clinical information such as smoking and gender with a radiomics model resulted in better predictive performance than the radiomics model alone [22]. Although several studies concluded that non-smoking women are more likely to have EGFR mutations [38-40], there is still no direct correlation between this clinical information and gene mutation status. 
According to the expert consensus in the latest NCCN guidelines, the mutually exclusive phenomenon widely exists among genetic mutations, especially between EGFR and KRAS genes [41-46]. Although the mechanism is not totally understood yet, one possible explanation for the mutually exclusive gene mutation phenomenon could be that tumors with KRAS mutations have already activated further downstream effectors and do not require EGFR mutations [41]. Based on the above information, the addition of mutually exclusive genetic information is more beneficial than other clinical information for optimizing the radiomics algorithm. This study confirms that mutually exclusive gene mutations can be used as additional useful clinical information to optimize radiomics algorithms for gene mutation prediction.

In clinical practice, most patients are tested for mutations in only a limited number of conventional genes such as EGFR. With the continuous addition of new targeted drugs such as KRAS-targeted sotorasib [11] and RET-targeted pralsetinib [13], the NCCN guidelines also point out the need for unconventional genetic testing in recent updates [47]. However, puncture biopsy can lead to risks such as pneumothorax, hemoptysis, and even death [48]. Our findings provide a non-invasive method other than repeat puncture biopsies to predict the status of new mutation targets and allow for more accurate prediction based on existing mutation results. This method not only has the potential to avoid secondary biopsies for patients at risk, but also may help to non-invasively guide the patient selection for new targeted drugs.

This study has some limitations. Firstly, this single center retrospective study has a relatively small number of KRAS mutation cases, because KRAS was not a routinely tested gene. Therefore, further validation in multi-center studies with expanded sample sizes is needed. Secondly, as research continues, more and more target genes for lung cancer are being discovered. By using EGFR and KRAS as examples, this study only validated our hypothesis that gene mutation mutual exclusion information has the potential to improve performance of radiomics algorithms, but more validation is required to test whether these results apply to other genes. Thirdly, the results of this study were obtained from imaging data and samples of primary lesions. Due to the heterogeneity of tumors, the mutation status may differ between the primary and metastatic lesions, or even within a single lesion [49]. Further studies are warranted to address more complex issues related to mutation heterogeneity.

\section{Declarations}

\section{Funding}

This work was supported by the National Natural Science Foundation of China (Grant Nos. 91959208, 81971646, 821220334, 81871379), the National Key Research and Development Program of China (Grant No. 2016YFC0103804), and the Natural Science Basic Research Program of Shaanxi Province (No. 2021JQ-352).

\section{Acknowledgements}


We would like to thank Dr. Zhoushe Zhao for his technical assistance.

\section{References}

1. Siegel RL, Miller KD, Jemal A. Cancer statistics, 2020. CA Cancer J Clin. 2020;70:7-30.

2. Sung H, Ferlay J, Siegel RL, Laversanne M, Soerjomataram I, Jemal A, et al. Global cancer statistics 2020: GLOBOCAN estimates of incidence and mortality worldwide for 36 cancers in 185 countries. CA Cancer J Clin. 2021;71:209-49.

3. Release Notice - Canadian Cancer Statistics. A 2020 special report on lung cancer. Health Promot Chronic Dis Prev Can. 2020;40:325.

4. Chen Z, Fillmore CM, Hammerman PS, Kim CF, Wong KK. Non-small-cell lung cancers: a heterogeneous set of diseases. Nat Rev Cancer. 2014;14:535-46.

5. Molina JR, Yang P, Cassivi SD, Schild SE, Adjei AA. Non-small cell lung cancer: epidemiology, risk factors, treatment, and survivorship. Mayo Clin Proc. 2008;83:584 - 94.

6. Shaw AT, Solomon BJ, Besse B, Bauer TM, Lin CC, Soo RA, et al. ALK resistance mutations and efficacy of lorlatinib in advanced anaplastic lymphoma kinase-positive non-small-cell lung cancer. J Clin Oncol. 2019;37:1370-9.

7. Solomon BJ, Besse B, Bauer TM, Felip E, Soo RA, Camidge DR, et al. Lorlatinib in patients with ALKpositive non-small-cell lung cancer: results from a global phase 2 study. Lancet Oncol. 2018;19:1654-67.

8. Drilon A, Oxnard GR, Tan DSW, Loong HHF, Johnson M, Gainor J, et al. Efficacy of selpercatinib in RET fusion-positive non-small-cell lung cancer. N Engl J Med. 2020;383:813-24.

9. National Comprehensive Cancer. Network (NCCN) guidelines for non-small cell lung cancer version 4.2021. https://www.nccn.org/guidelines/guidelines-detail?category=1\&id=1450.

10. Kerr KM, Bibeau F, Thunnissen E, Botling J, Ryška A, Wolf J, et al. The evolving landscape of biomarker testing for non-small cell lung cancer in Europe. Lung Cancer. 2021;154:161-75.

11. Sotorasib is active. in KRAS (G12C)-mutant non-small cell lung cancer. Cancer Discov. 2021;11:1870.

12. Reck M, Carbone DP, Garassino M, Barlesi F. Targeting KRAS in non-small-cell lung cancer: recent progress and new approaches. Ann Oncol. 2021;32:1101-10.

13. Cascetta P, Sforza V, Manzo A, Carillio G, Palumbo G, Esposito G, et al. RET inhibitors in non-smallcell lung cancer. Cancers (Basel). 2021;13.

14. Thein KZ, Velcheti V, Mooers BHM, Wu J, Subbiah V. Precision therapy for RET-altered cancers with RET inhibitors. Trends in cancer. 2021.

15. Kojadinovic A, Laderian B, Mundi PS. Targeting TRK. A fast-tracked application of precision oncology and future directions. Crit Rev Oncol Hematol. 2021;165:103451.

16. Yohe S, Thyagarajan B. Review of clinical next-generation sequencing. Arch Pathol Lab Med. 2017;141:1544-57. 
17. Mantilla WA, Sanabria-Salas MC, Baldion AM, Sua LF, Gonzalez DM, Lema M. NGS in lung, breast, and unknown primary cancer in Colombia: a multidisciplinary consensus on challenges and opportunities. JCO Glob Oncol. 2021;7:1012-23.

18. Santos M, Coudry RA, Ferreira CG, Stefani S, Cunha IW, Zalis MG, et al. Increasing access to nextgeneration sequencing in oncology for Brazil. Lancet Oncol. 2019;20:20-3.

19. Mu W, Jiang L, Zhang J, Shi Y, Gray JE, Tunali I, et al. Non-invasive decision support for NSCLC treatment using PET/CT radiomics. Nat Commun. 2020;11:5228.

20. Xi YB, Guo F, Xu ZL, Li C, Wei W, Tian P, et al. Radiomics signature: A potential biomarker for the prediction of MGMT promoter methylation in glioblastoma. J Magn Reson Imaging. 2018;47:13807.

21. Zhang J, Zhao X, Zhao Y, Zhang J, Zhang Z, Wang J, et al. Value of pre-therapy (18)F-FDG PET/CT radiomics in predicting EGFR mutation status in patients with non-small cell lung cancer. Eur $\mathrm{J}$ Nucl Med Mol Imaging. 2020;47:1137-46.

22. Wu G, Jochems A, Refaee T, Ibrahim A, Yan C, Sanduleanu S, et al. Structural and functional radiomics for lung cancer. Eur J Nucl Med Mol Imaging. 2021.

23. Weng Q, Hui J, Wang H, Lan C, Huang J, Zhao C, et al. Radiomic Feature-Based Nomogram: A Novel Technique to Predict EGFR-Activating Mutations for EGFR Tyrosin Kinase Inhibitor Therapy. Front Oncol. 2021;11:590937.

24. Ren M, Yang H, Lai Q, Shi D, Liu G, Shuang X, et al. MRI-based radiomics analysis for predicting the EGFR mutation based on thoracic spinal metastases in lung adenocarcinoma patients. Med Phys. 2021;48:5142-51.

25. Chang C, Zhou S, Yu H, Zhao W, Ge Y, Duan S, et al. A clinically practical radiomics-clinical combined model based on PET/CT data and nomogram predicts EGFR mutation in lung adenocarcinoma. Eur Radiol. 2021;31:6259-68.

26. Kim JO, Lee J, Shin JY, Oh JE, Jung CK, Park JK, et al. KIF5B-RET Fusion gene may coincide oncogenic mutations of EGFR or KRAS gene in lung adenocarcinomas. Diagn Pathol. 2015;10:143.

27. Mok T, Ladrera G, Srimuninnimit V, Sriuranpong V, Yu CJ, Thongprasert S, et al. Tumor marker analyses from the phase III, placebo-controlled, FASTACT-2 study of intercalated erlotinib with gemcitabine/platinum in the first-line treatment of advanced non-small-cell lung cancer. Lung Cancer. 2016;98:1-8.

28. Delbeke D, Coleman RE, Guiberteau MJ, Brown ML, Royal HD, Siegel BA, et al. Procedure guideline for tumor imaging with 18F-FDG PET/CT 1.0. J Nucl Med. 2006;47:885-95.

29. Kang F, Mu W, Gong J, Wang S, Li G, Li G, et al. Integrating manual diagnosis into radiomics for reducing the false positive rate of (18)F-FDG PET/CT diagnosis in patients with suspected lung cancer. Eur J Nucl Med Mol Imaging. 2019;46:2770-9.

30. Kang F, Wang S, Tian F, Zhao M, Zhang M, Wang Z, et al. Comparing the Diagnostic Potential of 68Ga-Alfatide II and 18F-FDG in Differentiating Between Non-Small Cell Lung Cancer and Tuberculosis. J Nucl Med. 2016;57:672-7.

Page $10 / 18$ 
31. Kang F, Han Q, Zhou X, Zheng Z, Wang S, Ma W, et al. Performance of the PET vascular activity score (PETVAS) for qualitative and quantitative assessment of inflammatory activity in Takayasu's arteritis patients. Eur J Nucl Med Mol Imaging. 2020;47:3107-17.

32. Han Y, Ma Y, Wu Z, Zhang F, Zheng D, Liu X, et al. Histologic subtype classification of non-small cell lung cancer using PET/CT images. Eur J Nucl Med Mol Imaging. 2021;48:350-60.

33. Zhou Y, Ma XL, Zhang T, Wang J, Zhang T, Tian R. Use of radiomics based on (18)F-FDG PET/CT and machine learning methods to aid clinical decision-making in the classification of solitary pulmonary lesions: an innovative approach. Eur J Nucl Med Mol Imaging. 2021;48:2904-13.

34. Liu Q, Sun D, Li N, Kim J, Feng D, Huang G, et al. Predicting EGFR mutation subtypes in lung adenocarcinoma using (18)F-FDG PET/CT radiomic features. Transl Lung Cancer Res. 2020;9:54962.

35. Yu Z, Ji H, Xiao J, Wei P, Song L, Tang T, et al. Predicting adverse drug events in Chinese pediatric inpatients with the associated risk factors: a machine learning study. Front Pharmacol. 2021;12:659099.

36. Zhang M, Tong E, Wong S, Hamrick F, Mohammadzadeh M, Rao V, et al. Machine learning approach to differentiation of peripheral schwannomas and neurofibromas: a multi-center study. Neuro Oncol. 2021.

37. Koyasu S, Nishio M, Isoda H, Nakamoto $Y$, Togashi K. Usefulness of gradient tree boosting for predicting histological subtype and EGFR mutation status of non-small cell lung cancer on (18)F FDG-PET/CT. Ann Nucl Med. 2020;34:49-57.

38. Dogan S, Shen R, Ang DC, Johnson ML, D'Angelo SP, Paik PK, et al. Molecular epidemiology of EGFR and KRAS mutations in 3,026 lung adenocarcinomas: higher susceptibility of women to smokingrelated KRAS-mutant cancers. Clin Cancer Res. 2012;18:6169-77.

39. Lynch TJ, Bell DW, Sordella R, Gurubhagavatula S, Okimoto RA, Brannigan BW, et al. Activating mutations in the epidermal growth factor receptor underlying responsiveness of non-small-cell lung cancer to gefitinib. N Engl J Med. 2004;350:2129-39.

40. Shi Y, Au JS, Thongprasert S, Srinivasan S, Tsai CM, Khoa MT, et al. A prospective, molecular epidemiology study of EGFR mutations in Asian patients with advanced non-small-cell lung cancer of adenocarcinoma histology (PIONEER). J Thorac Oncol. 2014;9:154-62.

41. National Comprehensive Cancer. Network (NCCN) guidelines for non-small cell lung cancer version 5.2021. https://www.nccn.org/guidelines/guidelines-detail? category=1\&id=1450.

42. Kosaka T, Yatabe Y, Endoh H, Kuwano H, Takahashi T, Mitsudomi T. Mutations of the epidermal growth factor receptor gene in lung cancer: biological and clinical implications. Cancer Res. 2004;64:8919-23.

43. Sequist LV, Heist RS, Shaw AT, Fidias P, Rosovsky R, Temel JS, et al. Implementing multiplexed genotyping of non-small-cell lung cancers into routine clinical practice. Ann Oncol. 2011;22:261624. 
44. Marchetti A, Martella C, Felicioni L, Barassi F, Salvatore S, Chella A, et al. EGFR mutations in nonsmall-cell lung cancer: analysis of a large series of cases and development of a rapid and sensitive method for diagnostic screening with potential implications on pharmacologic treatment. J Clin Oncol. 2005;23:857-65.

45. Roosan MR, Mambetsariev I, Pharaon R, Fricke J, Husain H, Reckamp KL, et al. Usefulness of Circulating Tumor DNA in Identifying Somatic Mutations and Tracking Tumor Evolution in Patients With Non-small Cell Lung Cancer. Chest. 2021;160:1095-107.

46. Amado RG, Wolf M, Peeters M, Van Cutsem E, Siena S, Freeman DJ, et al. Wild-type KRAS is required for panitumumab efficacy in patients with metastatic colorectal cancer. J Clin Oncol. 2008;26:162634.

47. Arteaga CL. Overview of epidermal growth factor receptor biology and its role as a therapeutic target in human neoplasia. Semin Oncol. 2002;29:3-9.

48. Cerci JJ, Bogoni M, Cerci RJ, Masukawa M, Neto CCP, Krauzer C, et al. PET/CT-guided biopsy of suspected lung lesions requires less rebiopsy than CT-guided biopsy due to inconclusive results. $\mathrm{J}$ Nucl Med. 2021;62:1057-61.

49. Zhou H, Hu Y, Luo R, Zhao Y, Pan H, Ji L, et al. Multi-region exome sequencing reveals the intratumoral heterogeneity of surgically resected small cell lung cancer. Nat Commun. 2021;12:5431.

\section{Tables}

Due to technical limitations, table 1 to 4 is only available as a download in the Supplemental Files section.

\section{Figures}


a

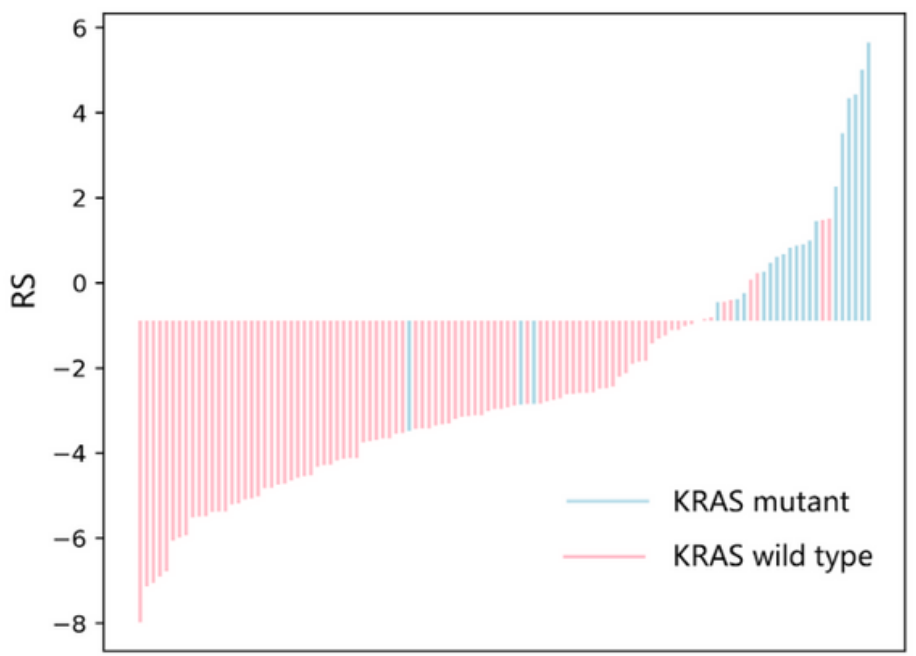

C

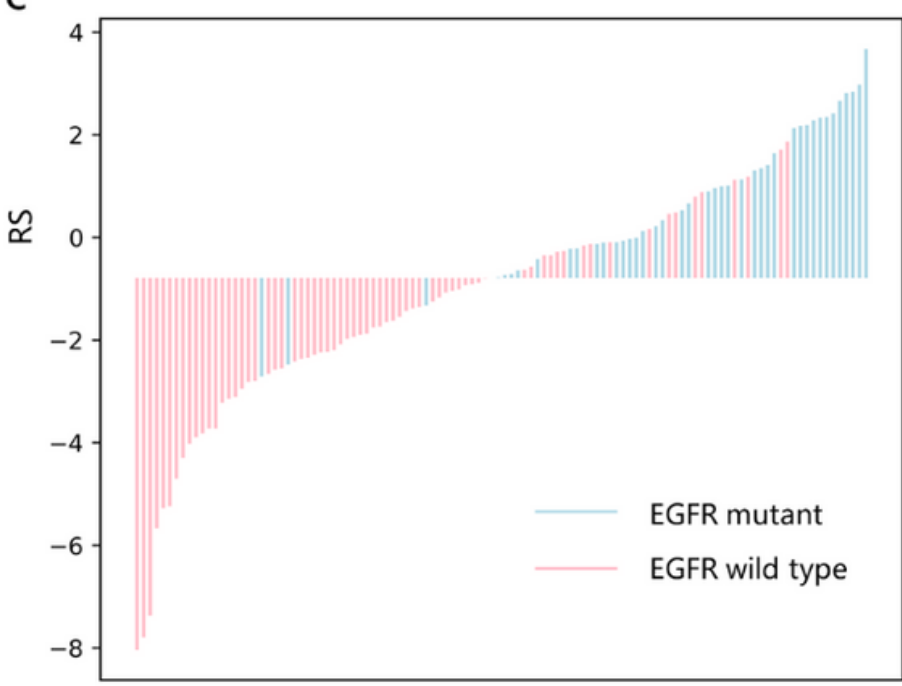

b

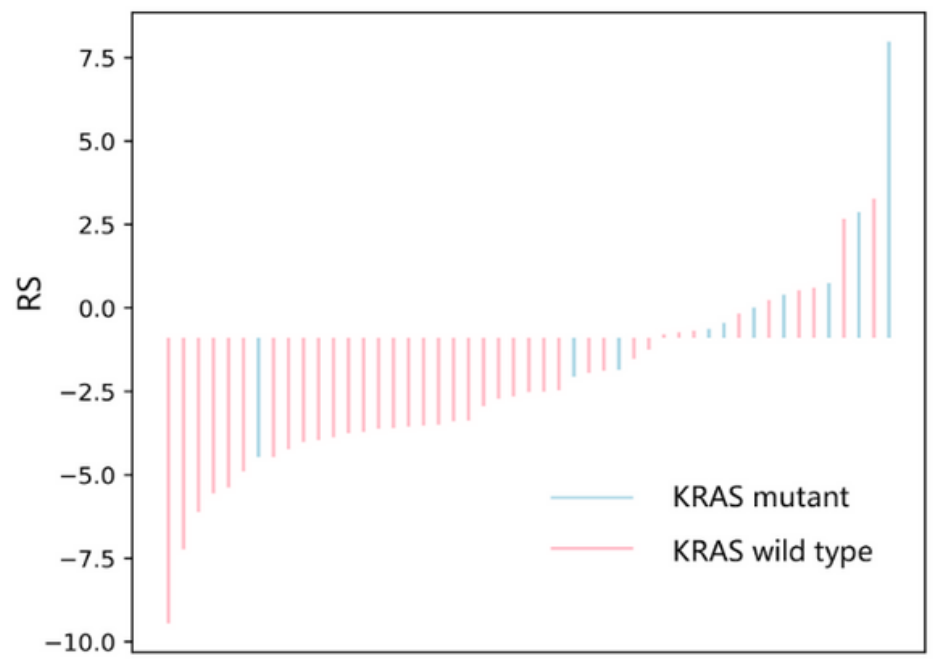

$\mathrm{d}$

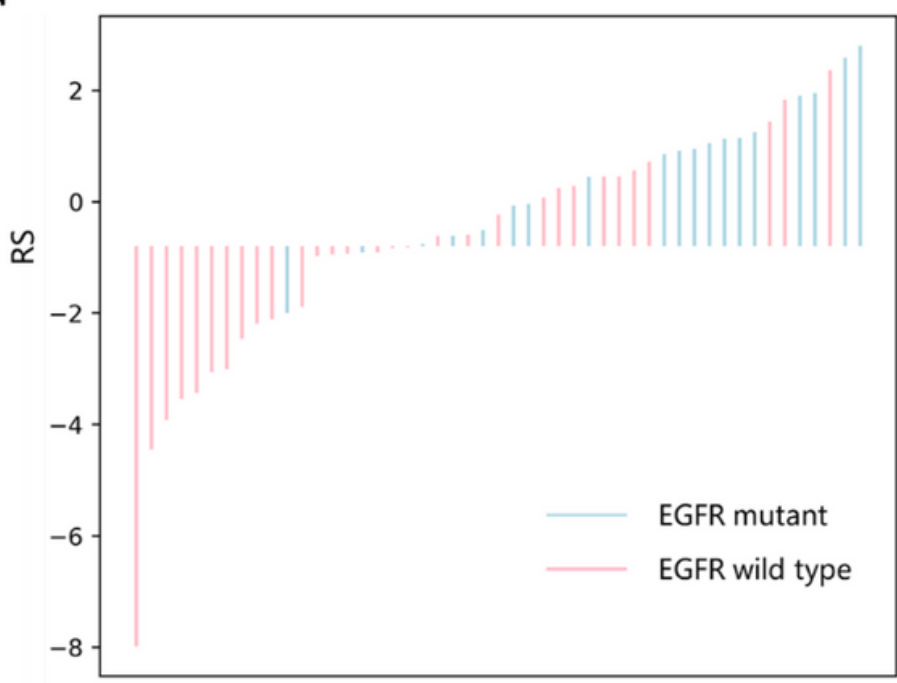

\section{Figure 1}

a RS for each patient in the training cohort in predicting KRAS mutations. $b$ RS for each patient in the test cohort in predicting KRAS mutations. c RS for each patient in the training cohort in predicting EGFR mutations. $\mathrm{d}$ RS for each patient in the test cohort in predicting EGFR mutations. 
a

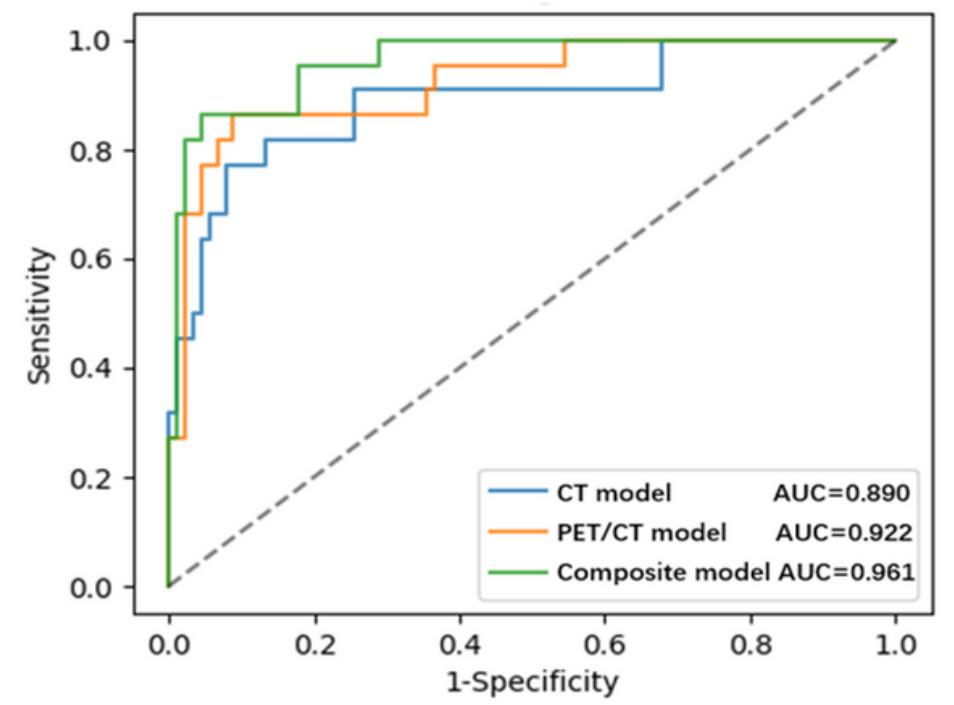

\section{C}

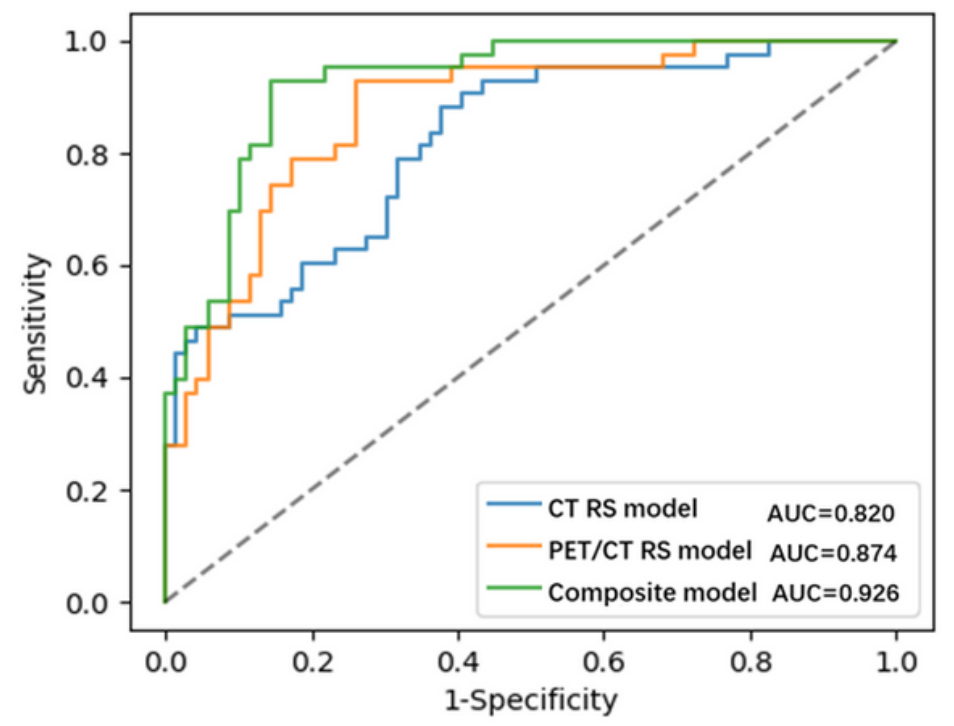

b

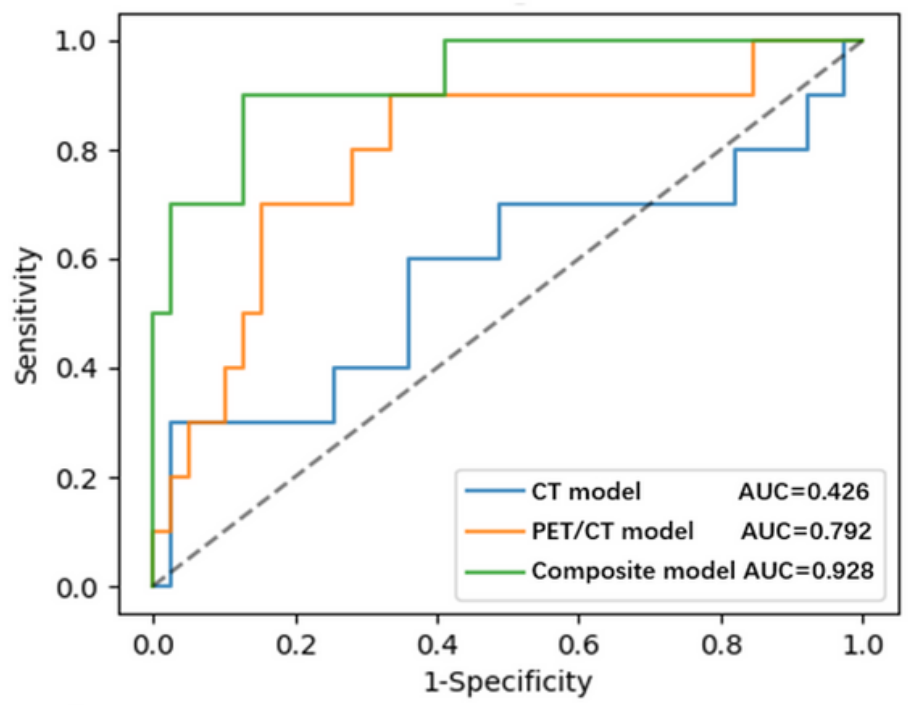

d

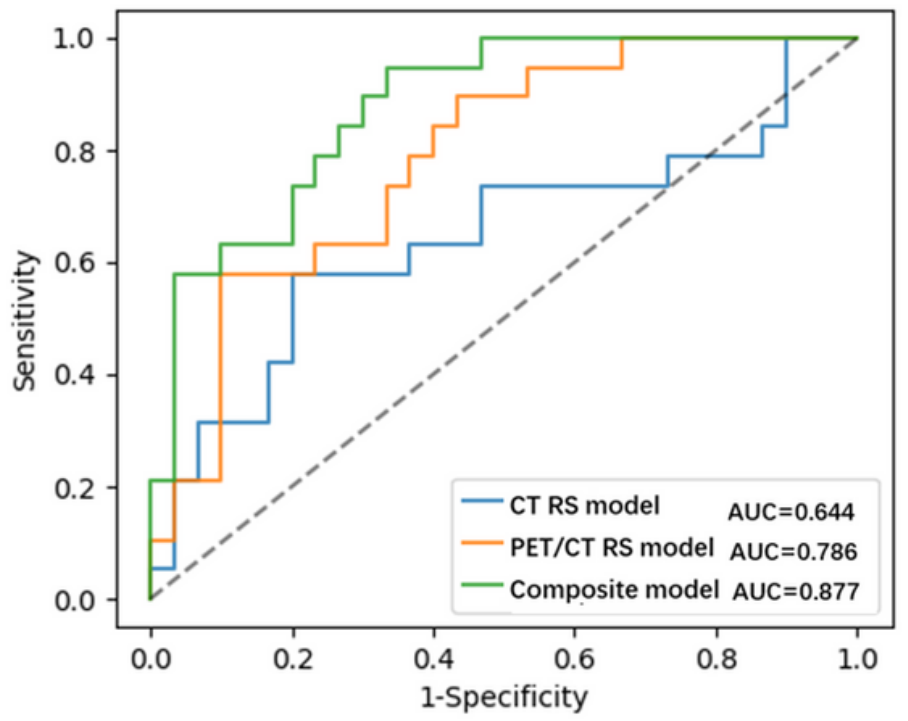

Figure 2

ROC curves of the three models. a The ROC curve of the training cohort for predicting KRAS mutations. $b$ The ROC curve of the test cohort for predicting KRAS mutations. c The ROC curve of the training cohort for predicting EGFR mutations. $d$ The ROC curve of the test cohort for predicting EGFR mutations. 
a

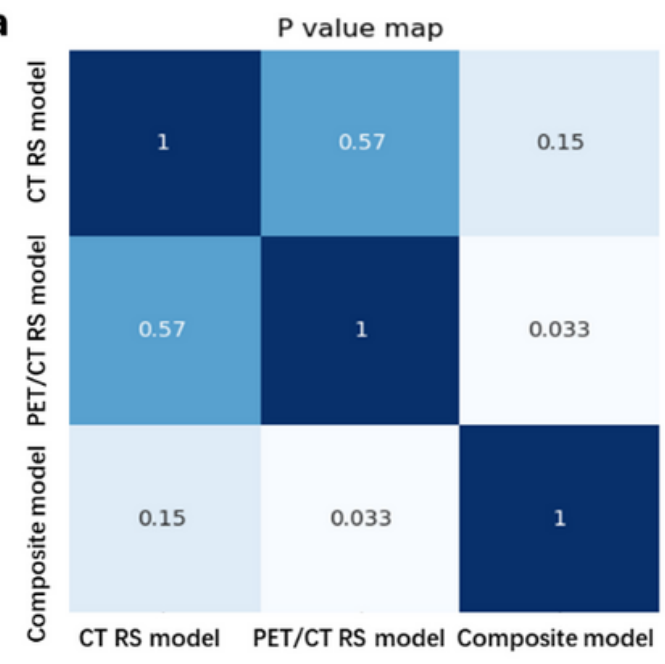

$$
\text { c }
$$

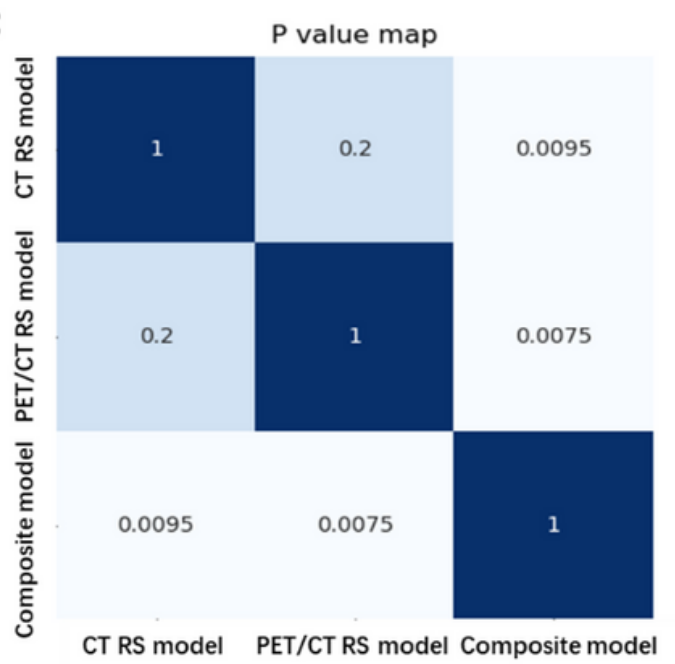

b
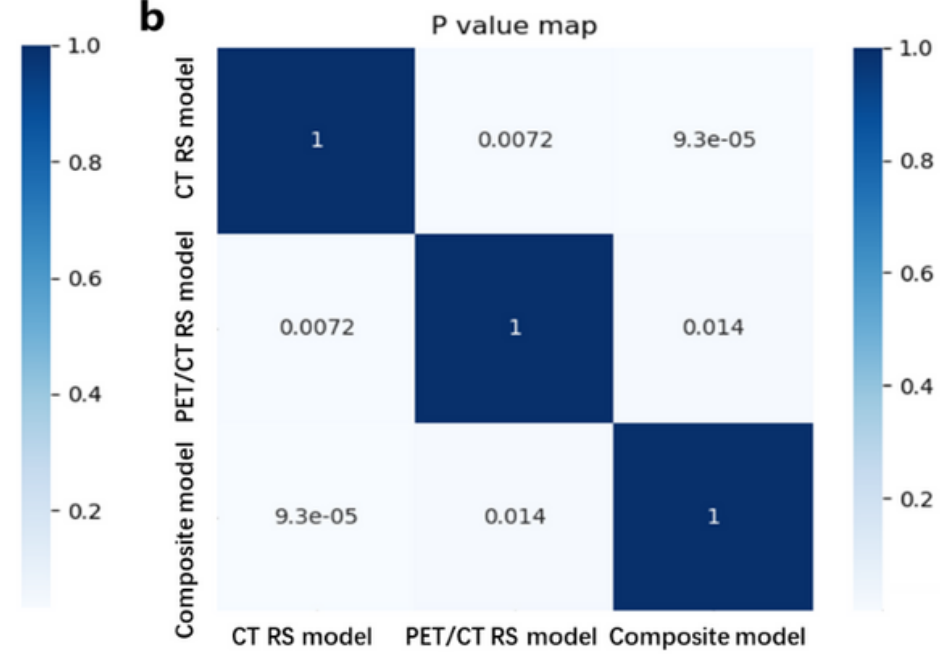

d

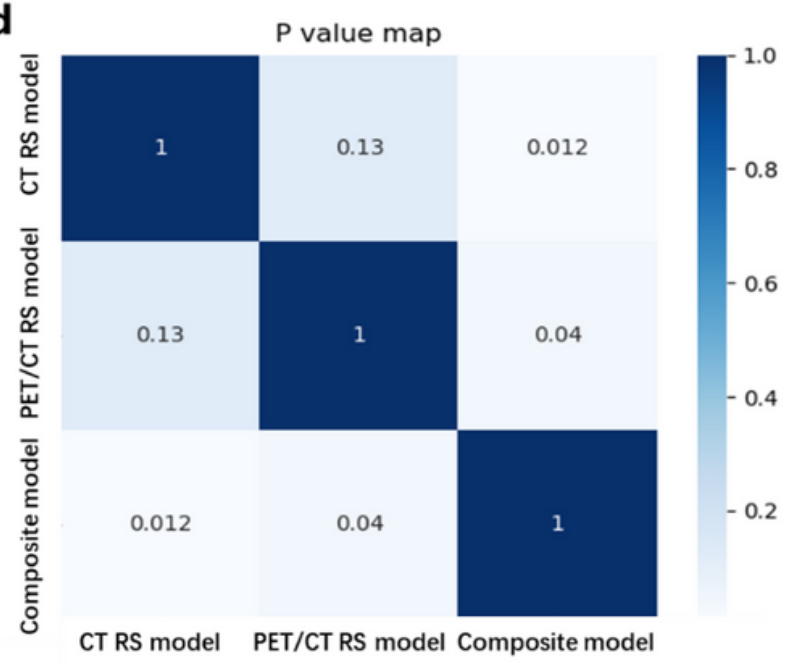

\section{Figure 3}

$P$ value maps of the three models. a The $P$ value map of the training cohort for predicting KRAS mutations. $b$ The $P$ value map of the test cohort for predicting KRAS mutations. $c$ The $P$ value map of the training cohort for predicting EGFR mutations. $d$ The $P$ value map of the test cohort for predicting EGFR mutations. 
a

Points

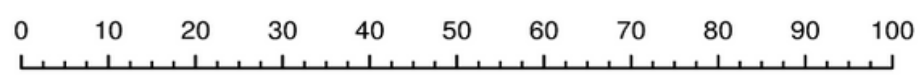

RS

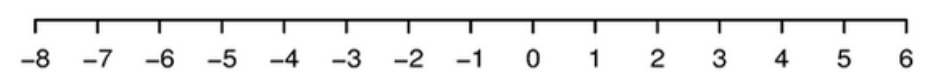

EGFR

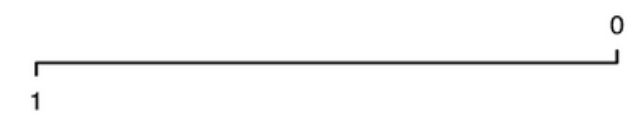

Total Points

\begin{tabular}{llllllllll}
\hline & 20 & 40 & 60 & 80 & 100 & 120 & 140 & 160 & 180
\end{tabular}

Risk

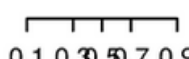

b

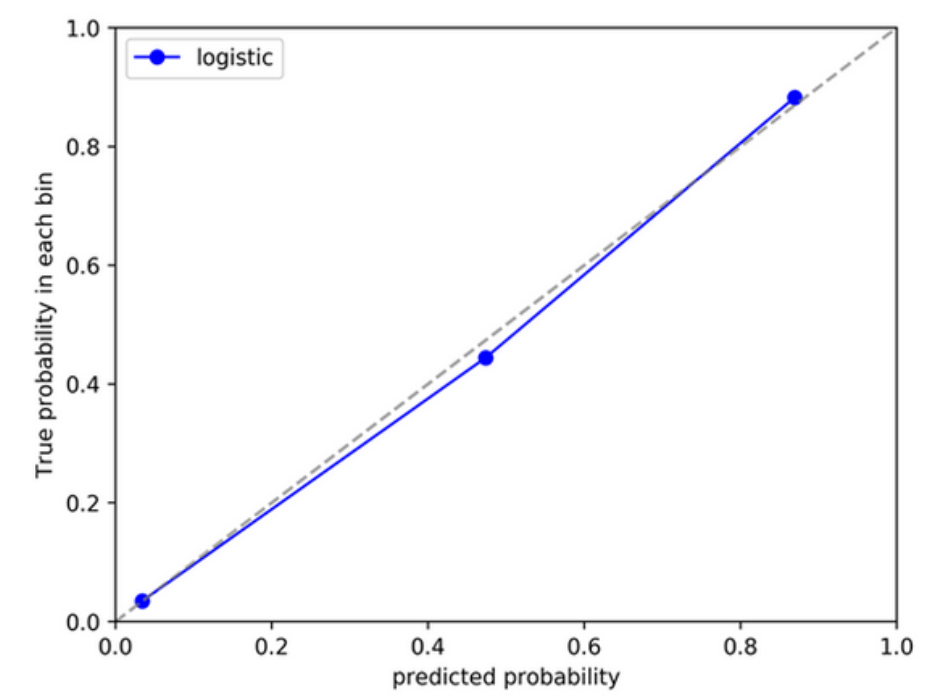

C

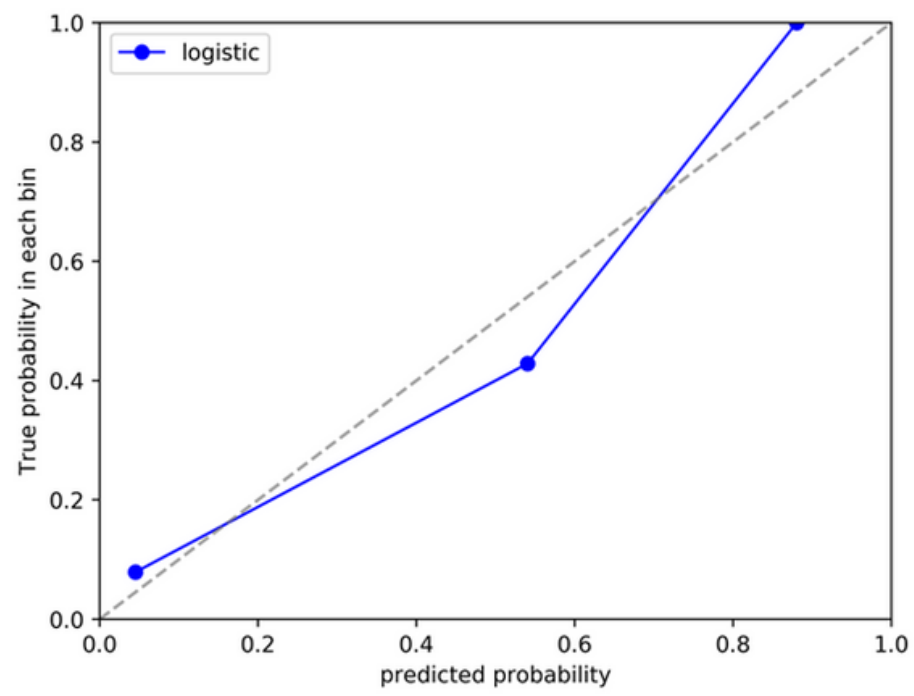

Figure 4

a Nomogram establishment by integrating PET/CT RS and EGFR. b, c Nomogram calibration curves in the training and test cohorts. The diagonal dashed line represents a predicted value equal to the true value, and the solid blue line is the model's prediction of KRAS mutation. The closer the two lines are, the better the performance. 
a

Points

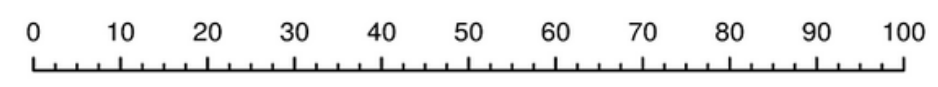

RS

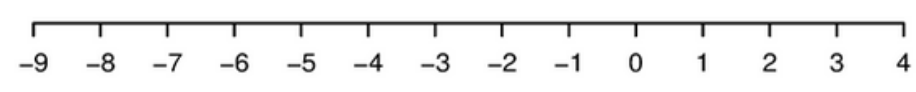

KRAS

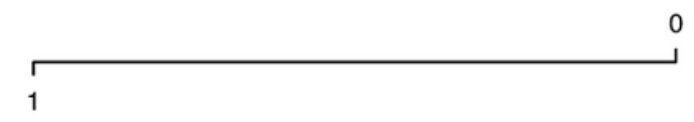

Total Points

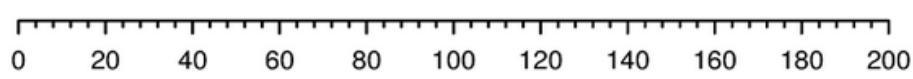

Risk

b

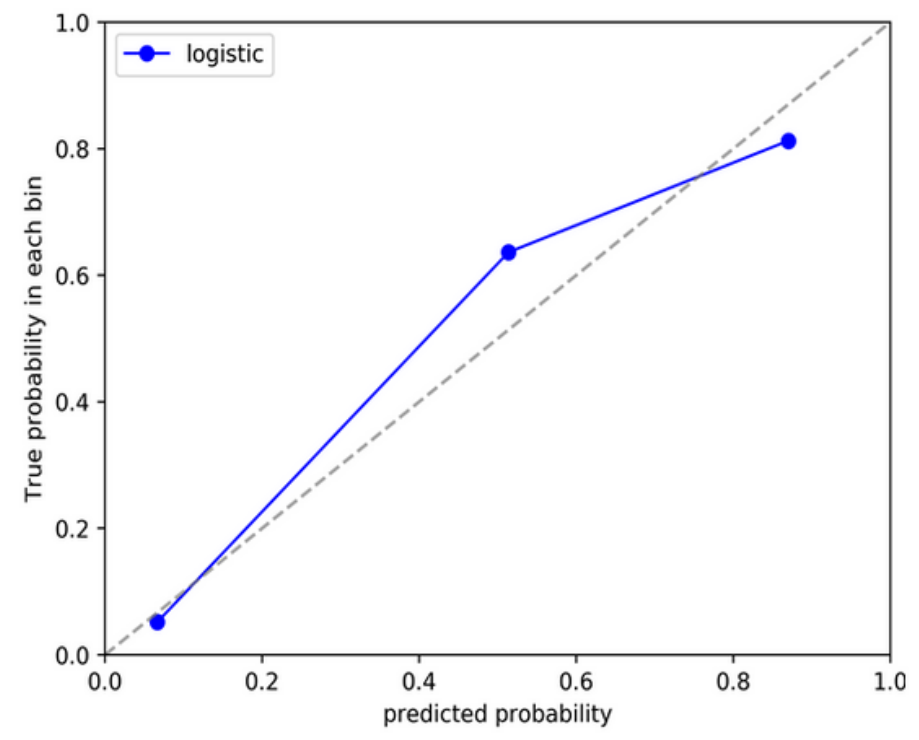

C

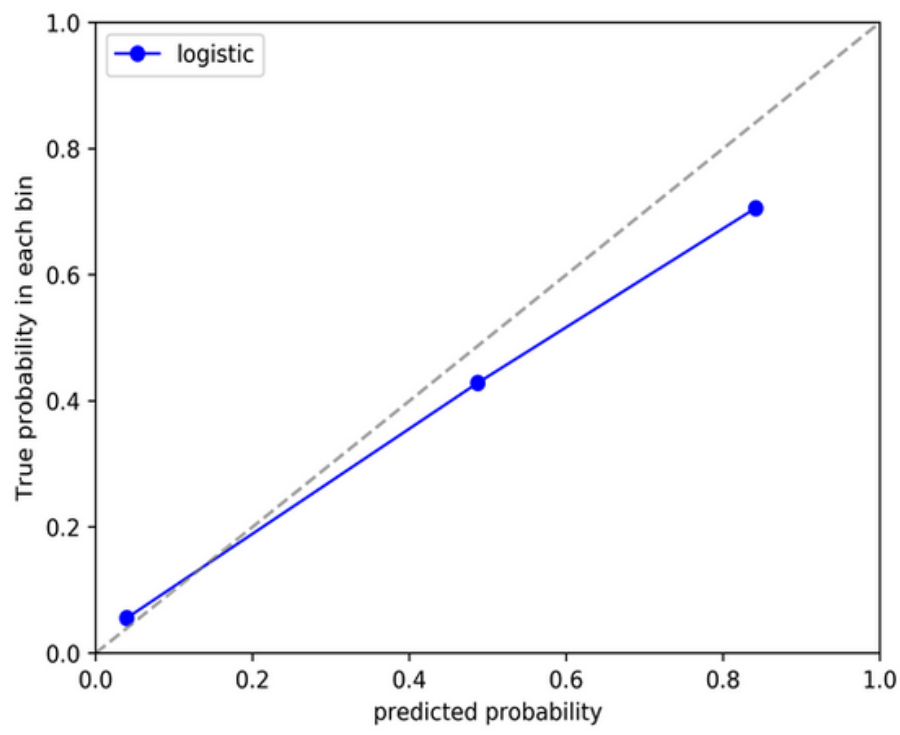

\section{Figure 5}

a Nomogram establishment by integrating PET/CT RS and KRAS. b, c Nomogram calibration curves in the training and test cohorts. The diagonal dashed line represents a predicted value equal to the true value, and the solid blue line is the model's prediction of EGFR mutation. The closer the two lines are, the better the performance. 

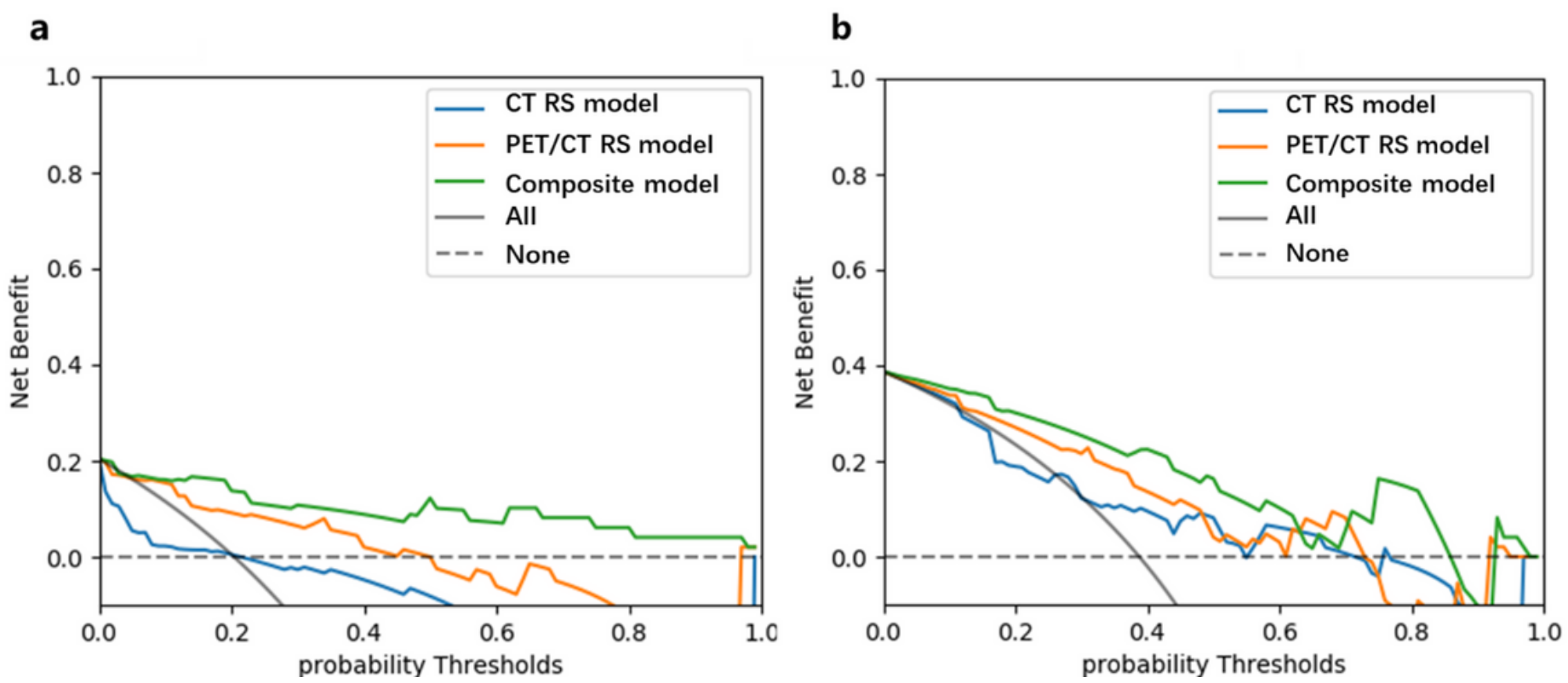

\section{Figure 6}

Decision curves of the three models. The green line represents the composite model incorporating PET/CT RS and mutually exclusive gene information. The blue and orange lines represent CT and PET/CT models, respectively. The grey line indicates the assumption that all patients possess the gene mutation, while the black dashed line indicates the assumption that all patients possess the wild-type gene. a Predicting KRAS mutation. b Predicting EGFR mutation.

\section{Supplementary Files}

This is a list of supplementary files associated with this preprint. Click to download.

- Table.docx

- SupplementaryMaterials.docx 